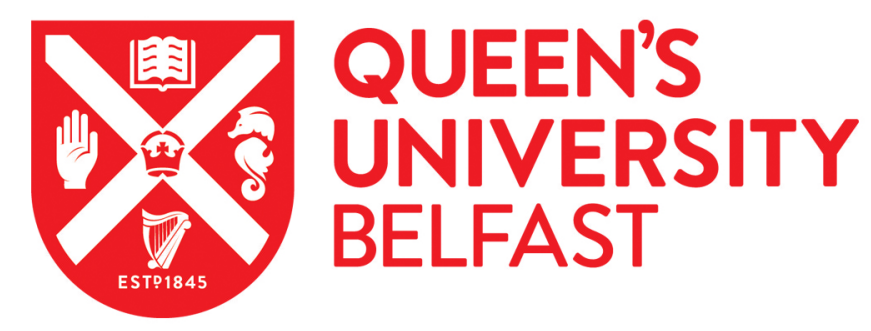

\title{
Power System Inertia Estimation using HVDC Power Perturbations
}

Best, R. J., Brogan, P. V., \& Morrow, D. J. (2021). Power System Inertia Estimation using HVDC Power Perturbations. IEEE Transactions on Power Systems, 36(3), 1890-1899.

https://doi.org/10.1109/TPWRS.2020.3028614

\section{Published in:}

IEEE Transactions on Power Systems

\section{Document Version:}

Peer reviewed version

Queen's University Belfast - Research Portal:

Link to publication record in Queen's University Belfast Research Portal

\section{Publisher rights}

(C) 2020 IEEE.

This work is made available online in accordance with the publisher's policies. Please refer to any applicable terms of use of the publisher.

\section{General rights}

Copyright for the publications made accessible via the Queen's University Belfast Research Portal is retained by the author(s) and / or other copyright owners and it is a condition of accessing these publications that users recognise and abide by the legal requirements associated with these rights.

Take down policy

The Research Portal is Queen's institutional repository that provides access to Queen's research output. Every effort has been made to ensure that content in the Research Portal does not infringe any person's rights, or applicable UK laws. If you discover content in the Research Portal that you believe breaches copyright or violates any law, please contact openaccess@qub.ac.uk. 


\title{
Power System Inertia Estimation using HVDC Power Perturbations
}

\author{
Robert J. Best, Paul V. Brogan and D. John Morrow
}

\begin{abstract}
This paper describes a power system inertia estimation method that utilizes power perturbations caused by the flow switch on an HVDC interconnector. The analysis is performed using real power system measurements from Ireland's all-island power system. The method employs an energy based estimation technique which permits a non-step power change to be used for inertia calculation and encapsulates the challenges associated with the use of real data. While it is shown that synchronous generators account for most of the power system inertia, other contributors are also investigated, namely power station auxiliary load, distributed generation and power system demand. Time-of-day and wind related effects on Ireland's power system inertia are explored. Recommendations are made for the practical implementation of the method.
\end{abstract}

Index Terms-- Frequency transients, Low inertia power systems, Phasor measurement data, Power system inertia

\section{INTRODUCTION}

$\mathrm{T}$ HE kinetic energy stored in the rotating masses of bulk synchronous generators provides most of the power system's inertia. As increasing penetrations of non-synchronous renewables displace bulk synchronous generators, the inertia contribution from other sources, such as motors and distributed synchronous or induction generators, becomes more important. For example, on Ireland's all-island power system, a system nonsynchronous penetration (SNSP) of $75 \%$ is targeted for 2020 [1]. Improved real-time inertia estimation is essential for low inertia power system operation in terms of unit commitment and dispatching system services to ensure power system stability.

Synchronous inertial response is now a remunerated system service [2]. Improved estimation of inertia can help determine how much of this service should be bought and how to appropriately scale its value when the resource is scarce [3]. Thus, in addition to its technical necessity there is a strong financial impetuous for system inertial like services, and further, this necessitates the verification of response from both synchronous and synthetic inertia providers.

Many methods of inertia calculation rely on power system frequency transients caused by loss of generation [4] - [9]. Such loss of generation events are relatively rare and although they tend to cause large frequency disturbances they require a post event study to determine cause, location, type of trip, etc., so that inertia and system response can be characterized. The scarcity of these events, in particular repeated similar events, makes validation of these methodologies difficult.

Techniques that provide online estimation of inertia have also been proposed [10], [11]. These methods utilize ambient power system changes, wide-area measurements and sophisticated algorithms to achieve online inertia estimation. It is likely that the most accurate method will result from a combination of the various techniques that have been developed. The major difference between these techniques tends to relate to the type of monitoring data that is available, or required, as an input. This could range from a single frequency measurement and knowledge of the tripped unit's output, to power and frequency measurement at every infeed and major load. In addition, there is the difficulty in acquiring data for field testing of techniques.

The method proposed in this paper is to observe a known active power perturbation, then by accurately monitoring power system frequency measurements use dynamic equations to estimate system inertia. The case study explored occurs when the power flow over an HVDC interconnector switches direction, resulting in a $30.5 \mathrm{MW}$ step in power flow. The HVDC interconnector connects the Great Britain (GB) and the all-island power systems with reverses in power flow occurring regularly, largely driven by load and the generation mix between thermal and renewable. Although from the perspective of this study the change in HVDC power flow is a normal system operational event, such a perturbation can be injected by a battery energy storage system or HVDC link for the specific purpose of inertia estimation. A similar method is being used in Japan and is intended for GB [12], in this case a dedicated device injects power and uses a patented method to estimate power system inertia. The method demonstrated in this paper is in the public domain and utilizes an existing power system perturbation.

Probing methods of inertia estimation, whether controlled [12] or, as in this investigation, ambient can directly inform power system operators, but frequency and time series measurements can also be used to improve statistical estimations, such as [13]. It is worth noting that such statistical investigations and estimations may require probes of differing magnitudes, at different frequencies and with various generation

This work is part of SPIRE2 (Storage Platform for the Integration of Renewable Energy 2) project, supported by the European Union's INTERREG VA Programme, managed by the Special EU Programmes Body (SEUPB). The views and opinions expressed in this paper do not necessarily reflect those of the European Commission or SEUPB.

R. J. Best, P. V. Brogan and D. J. Morrow are with the School of EEECS, Queen's University Belfast, Northern Ireland, United Kingdom, (e-mail: r.best@qub.ac.uk). 
mixes if they are to remain accurate [14] especially as wind plant implement frequency response [15]. In this investigation frequency deviation was small and within the dead-band, maximizing the accuracy of the synchronous inertia and minimizing contamination from generator frequency response.

The analysis begins by modelling the power switch using a Matlab power system simulation. Although repeated with high consistency, the HVDC perturbation is complex and not a simple step change. The inertia estimation technique is developed with a focus on the challenges associated with the real power system data. The method needs to take into account the relatively small frequency disturbances, background effects on the load-demand balance, sources of inaccuracy or noise in the recorded Phasor Measurement Unit (PMU) data and the availability of power system information. The inertia estimation method is then applied to a data set of real events captured during 2015.

The swing equation is the basis of most estimates of power system inertia, with [16] providing a good example; however this analysis uses simulated data with a very low noise component. When dealing with real data it is common to employ filters, such as those discussed in [13] - [15]. In this investigation the integration of power with respect to time to give energy delivered is employed as a method to smooth the signal; logic is then applied to make an estimate of power system frequency. The results are compared to an estimate of power system synchronous inertia, based on unit commitment and machine parameters.

These power system inertia estimates are compared with other power system data, such as wind generation, demand and synchronous generator inertia. This allows a decomposition of the power system inertia sources to be performed. Lumped averages of system inertia and other event metrics are used to analyze how the inertia varies with time-of-day and the penetration of wind generation. The paper concludes with recommendations for how a real time implementation of this technique could be implemented in practice. One of the challenges associated with power system estimation is that at very low inertias the frequency estimation algorithms may not be able to keep pace with the transient response. A solution that might permit the method proposed in this paper to be applied more universally would be to complement frequency measurements with machine speed taken from rotary encoders.

\section{DeVelopment of Methodology}

Classically the understanding of power system inertia begins with the rotational kinetic energy of the machines connected to the power system. Expressed in (1) is the cumulative rotational energy of the power system, based on the rotational kinetic energy of each component. In reality some rotating machines are significant and easy to monitor, such as synchronous generators at power stations, but in principle every motor connected to the power system contributes to system inertia. When estimating system inertia from unit commitment of bulk synchronous generation $E_{S G}$ is estimated from the summation in (1). However, this method underestimates inertia as $N$ is small relative to the number of connected rotational devices.

$$
E_{S G}=\sum_{n=1}^{n=N} \frac{1}{2} \cdot J_{n}\left(2 \cdot \pi \cdot f_{n}\right)^{2}
$$

$E_{S G}$ is total rotational energy of synchronous generation in the system, $n$ is the rotational device identifier, $N$ is the number of machines synchronized, $J_{n}$ is the moment of inertia of the connected device and $f_{n}$ is the momentary frequency measured at the connected device. If $N$ were the total number of connected devices then the summation would yield the total rotational kinetic energy in the power system, $E_{s y s}$. It is not possible to do this practically, but as will be seen $E_{s y s}$ can be estimated.

Often it is assumed that power system frequency is universal across a system, especially on a small system such as Ireland. However, power systems are more appropriately modelled as a spring mass system, with each rotating machine applying torque on each other, proportional to phase angle change. This complex model is necessary following a disturbance; however, a theoretical position exists in the dynamic system, referred to as the center of inertia, about which fluctuations take place. With sufficient observability this center of inertia can be identified, measured or determined. The center of inertia will have the unperturbed system frequency and constant angular momentum. If a system is sufficiently rigid or considered over a sufficient time period, then the frequency at any given point $\left(f_{n}\right)$ will tend to the center of inertia frequency, $f$, and can be considered as being constant between connected devices. The change in kinetic energy $\Delta E$ is the difference between kinetic energy at frequency $f_{a}$ and $f_{b}$ due to a disturbance and (1) can be expressed as (2).

$$
\Delta E=\left(f_{a}{ }^{2}-f_{b}{ }^{2}\right) \sum_{n=1}^{n=N} \frac{1}{2} \cdot J_{n}(2 \cdot \pi)^{2}
$$

However, $\left(f_{a}{ }^{2}-f_{b}{ }^{2}\right)=\left(f_{a}-f_{b}\right)\left(f_{a}+f_{b}\right)$. If the frequency deviation is small and the starting frequency is close the nominal frequency of the system, $f_{0}$, then $f_{a} \approx f_{b} \approx f_{0}$ leading to (3) which can be substituted into (2). $\Delta f$ is the frequency deviation. Note that the error introduced by this assumption is negligible for the frequency transients analyzed in this paper.

$$
\left(f_{a}{ }^{2}-f_{b}{ }^{2}\right) \approx\left(f_{a}-f_{b}\right)\left(2 f_{0}\right)=\Delta f \cdot 2 f_{0}
$$

The energy perturbation $\Delta E$ arises from the power change measured by the PMU at the HVDC interconnector, this can be expressed with an integral between time $t=0$ and $t=T$ as on the left-hand side of (4), where $P_{t}$ is the disturbance power at time $t$. The right-hand side of (2) can be further simplified in (4) if all moments of inertia are considered as a summed quantity, equating to what would be measured at the center of inertia. $J$ is the total power system moment of inertia.

$$
\Delta E=\int_{t=0}^{t=T}\left(P_{t}-P_{t=0}\right) \cdot d t=4 \cdot \Delta f \cdot f_{0} \cdot J \cdot \pi^{2}
$$

Power system engineers tend to express the inertia of a power system as the energy stored in the rotating masses, typically using a unit such as Megawatt-seconds (MWs). Angular momentum is related to stored energy via (5). 


$$
E_{s y s}=\frac{1}{2} J \omega_{0}^{2}=\frac{1}{2} J\left(2 \cdot \pi \cdot f_{0}\right)^{2}
$$

$E_{s y s}$ is the total rotational energy of the power system at nominal frequency and angular velocity, $\omega_{0}$.

Substituting (5) into (4) gives (6).

$$
\int_{t=0}^{t=T}\left(P_{t}-P_{t=0}\right) \cdot d t=2 \cdot \frac{\Delta f}{f_{0}} E_{s y s}
$$

The method is developed with the contention that any active power perturbation should be relatively small compared to system demand so that inertia characterization could be performed without frequency deviating beyond statutory limits. The ideal case would involve the application of a step change in power sufficient to cause a frequency disturbance, while not being disruptive. The frequency would then be measured to calculate rate-of-change-of-frequency (ROCOF) as soon as possible following the load change, before generator governor response and other power system interactions occur. In this scenario, for small frequency deviations the rotational kinetic energy, expressed as Megawatt-seconds (MWs), and ROCOF would be directly proportional. However, this is complicated by several factors, such as the difficulty in dynamically measuring frequency from voltage waveforms, particularly immediately after a nonlinearity [17].

The real case study will provide an understanding of the associated difficulties and limitations of power system inertia estimation. It so happens that the switching of power direction by the HVDC interconnector produces a very repeatable power characteristic, however, it is not a step change.

\section{A. Observation of power flow direction switch}

The power during a typical switch of direction from import to export on the HVDC link is shown in Fig. 1. The process takes approximately 1 second and results in a power change of $30.5 \mathrm{MW}$. There is negligible variation between occurrences, as Fig. 1 actually shows 20 such events superimposed. The change in power of $30.5 \mathrm{MW}$ represents in the region of $1 \%$ to $0.5 \%$ of the total system demand. The maximum credible outage in this power system is typically up to $450 \mathrm{MW}$, which would cause frequency nadirs of approximately 49.2 to $49.3 \mathrm{~Hz}$ with the grid's current operating conditions. Example frequency transients resulting from the HVDC direction switch are shown in Fig. 2. The frequency plot exhibits spikes around the parts of the transient with the most rapid changes in power. The frequency for these data has been measured at the HVDC interconnector and thus is more susceptible to noise and frequency estimation algorithm errors than measurements taken elsewhere or combined from multiple locations.

The power flow switch can occur in either direction; both 'to-export' and 'to-import' changes are considered in the analysis. A method to characterize inertia from these events is developed through simulation, in Matlab SimPowerSystems, and applied to the field data.

\section{B. Simulated Transients}

The method to characterize power system inertia begins with power system simulation. This simulation emphasizes the challenges associated with derivations from real power system data. A single bus network is deemed sufficient as the goal is a robust analysis of real power system data rather than simulation complexity. The model consists of a single turbine-generator, a system demand and a dynamic load to allow the application of the real power perturbation. System inertia and system droop are varied in the simulation. In the Irish power system synchronous inertia is dispatched by the system operator, using owner provided data for the dispatchable synchronous generators' inertia. There are other causes of variation of power system inertia in the grid, which will be discussed later. A range of $20000 \mathrm{MWs}$ to $60000 \mathrm{MWs}$ should encapsulate the full range of Irish power system inertia. During the period of data collection the minimum permitted inertia from synchronous generators was 20000 MWs, according to system operating constraints [18].

The effective droop of the power system depends on the units dispatched and their loading conditions. Many units may be either fully loaded or not capable of being dispatched to provide a droop response. In the Irish network a significant proportion of primary operating reserve can be provided by spinning pumped hydro and HVDC static reserve. Analysis of PMU data indicates that the delay in starting pumped hydro will be in excess of the key part of the frequency transient for inertia characterization [19]. Static reserve occurs at frequency set-points below the range of the analyzed frequency transients. This suggests that the effective droop can be expected to vary significantly. A range of $5 \%$ to $10 \%$ is applied to the simulation.

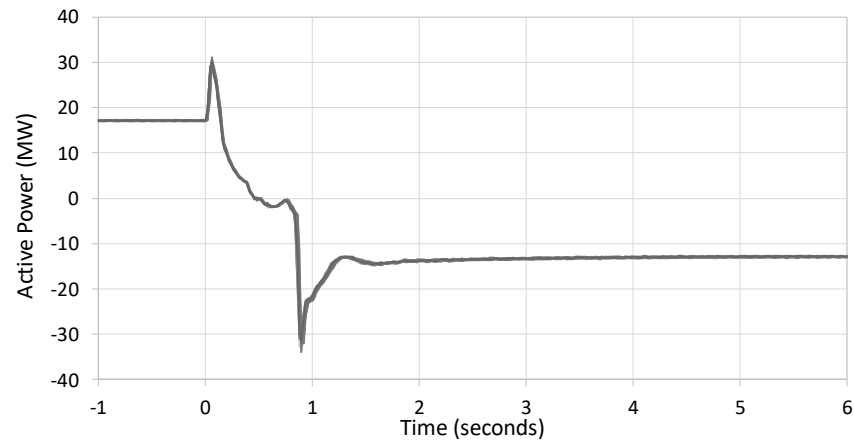

Fig. 1. Power flow switch on HVDC interconnector; 20 superimposed

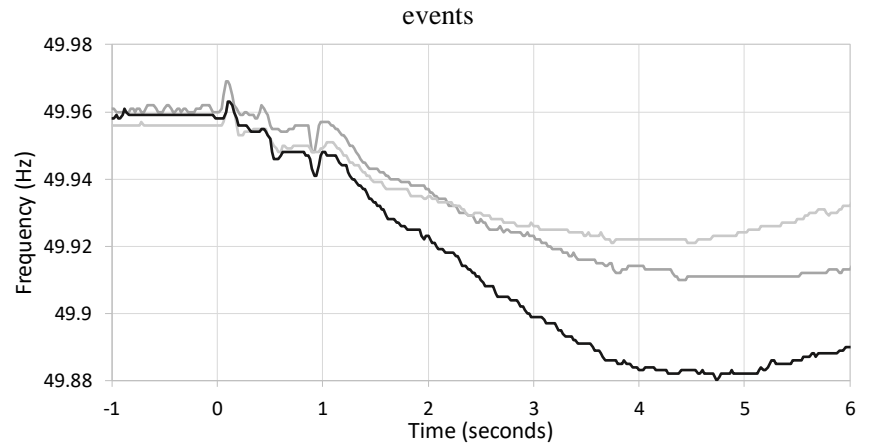

Fig. 2. Frequency transients caused by power flow switch

\section{Calculation of Inertia from Simulated transients}

Frequency traces from the simulation are presented in Fig. 3 for a number of combinations of inertia and droop. These are 
comparable with the measured data in Fig. 2 in terms of frequency nadir and time of nadir. The main difference is that the simulation is free from measurement effects, such as noise.

The system inertia can be determined from the simulation data using (7) which is a rearrangement of (6), where $E_{s y s}$ is made the subject of the equation, kinetic energy in MWs, $f_{0}$ is the nominal system frequency of $50 \mathrm{~Hz}, \frac{d f}{d t}$ is the ROCOF and $\Delta P$ is the instantaneous difference between the power perturbation transient and the generator governor response. Fig. 4 shows the inertia estimation using a 0.04 s moving average of ROCOF calculated from machine speed for two of the frequency transients in Fig. 3. The reason for the difference in the two plots is due to the more aggressive 5\% droop having an earlier nadir, see Fig. 3. It can be observed that an accurate inertia estimation should be possible, however, there will be difficulty achieving this during certain parts of the power transient. Most of these occur during the first second. After this, reasonable system inertia interpretation can be made until near to the frequency nadir, where the ROCOF and $\Delta P$ reduce to such small values that simulation error has significant effect.

$$
E_{\text {sys }}=\frac{f_{0}}{2} \Delta P /\left(\frac{d f}{d t}\right)
$$

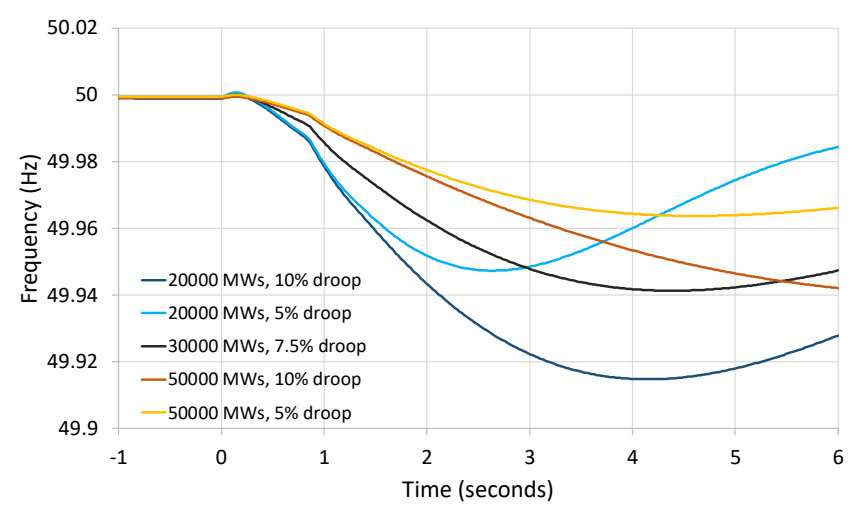

Fig. 3. Simulated frequency traces for various levels of droop and inertia

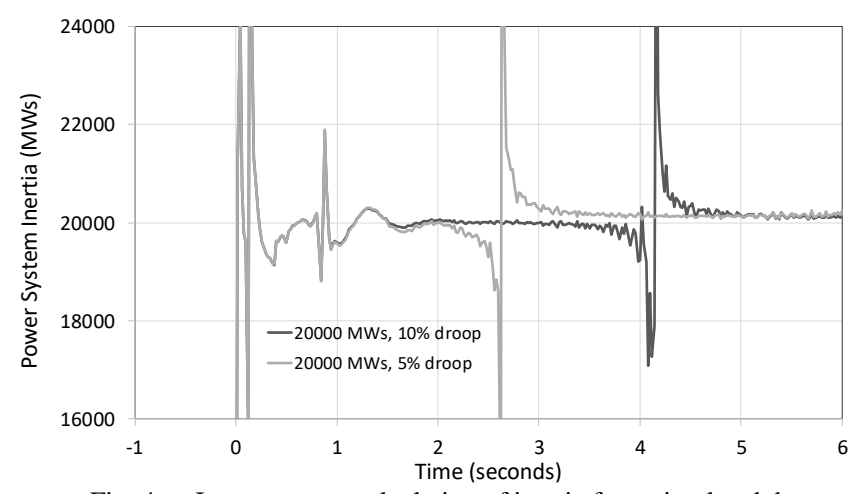

Fig. 4. Instantaneous calculation of inertia from simulated data

\section{ADAPTION OF INERTIA ESTIMATION FOR USE WITH GRID MEASUREMENTS}

For the practical implementation, the governor response will not be available, and even the measurement of active power from all generators would still require decomposition of the inertial and governor responses. In addition, the effective fre- quency response of the whole power system will include an element of under frequency load relief.

Not including load relief and governor response results in an increasing error with time, which would suggest limiting the analysis to the start of the transient. However, the simulation has highlighted that this approach may be problematic. It will be necessary to strike a balance when analyzing the real data.

An estimate of governor response and load relief, say 'power system response', if applied in a reasonable manner to the measured data would improve accuracy of calculation closer to the frequency nadir. A range of governor responses will occur across the suite of installed generators, which additionally depends on factors such as loading conditions, making it difficult to define a specific response characteristic. However, it is known that governor response and load relief to the perturbation is $0 \mathrm{MW}$ at $\mathrm{t}=0$, and that at the time of the frequency nadir the $\Delta P$ has reduced to zero, i.e governor response and load relief are equal to $30.5 \mathrm{MW}$. This will be estimated as a time delay plus a linearly rising system response. A more complex method could be applied but it would be difficult to determine if including generator turbine time constants, etc, would make this inertia estimation significantly more accurate.

Even if a reasonably accurate estimate of system response to the perturbation is applied to the calculation, this does not prevent other background variations in generation-load affecting the outcome. This is more likely to occur with increased time, thus although there is benefit in increasing the calculation window, the potential for error introduced by background variations will also increase.

\section{A. Frequency Estimation}

The frequency in the real data is estimated from voltage wave measurements and so it takes several cycles to calculate frequency with high confidence. Tests on the measurement equipment show that frequency takes 1 to 2 cycles longer to calculate than active power, particularly during dynamic conditions [19]. The accuracy of frequency estimation is severely reduced if calculated from data acquired during conditions that affect the voltage phase and magnitude. This is the case for several cycles after the rapid changes in power, i.e. close to $t=0$, and close to $\mathrm{t}=0.9 \mathrm{~s}$.

The frequency data is taken at the source of the perturbation which is not ideal, but sufficient data was not available from other locations. Voltage wave data at other locations would be less affected by the perturbation. This methodology could be further refined if frequency from multiple measurement locations were combined to more accurately estimate center of inertia frequency [20]; this would mitigate the effect of any intra or inter area oscillations.

Data resolution is $0.001 \mathrm{~Hz}$ and noise is evident, see Fig. 1. For this reason, the use of larger values of frequency deviation, that span a longer time period, will be important. Data will be averaged over several cycles (a 25 cycle moving average is used in this study) and calculation performed over as long a period as possible while taking into account other conflicting factors. 


\section{B. Inertia Estimation and Characterization}

For small frequency deviations close to the nominal operating frequency, the energy that has been extracted from the rotating masses is directly proportional to the frequency deviation. From this, the total rotating kinetic energy at nominal operating frequency can be calculated, which is often expressed in MWs and referred to as power system inertia. This is shown in (8), which is adapted from (7), where $P_{m}(t)$ is the power system response at time $t, P_{e}(t)$ is power change caused by the disturbance at time $t, \Delta f$ is the frequency difference between $t=a$ and $t=b$, and $\Delta E$ is the energy difference between $t=a$ and $t=b$.

$$
E_{\text {sys }}=\frac{f_{0}}{2} \int_{t=b}^{t=a}\left(P_{m}(t)-P_{e}(t)\right) d t / \Delta f=\frac{f_{0}}{2} \cdot \Delta E / \Delta f
$$

The energy change caused by the disturbance is calculated for each cycle from the start of the disturbance. The energy from power system response will be estimated as a time delay and linear slope of power, with any error in this estimate growing with increasing time. Fig. 5 shows the estimated energy extracted from the system against time, for a perturbation with a frequency nadir at 4.7 seconds. The plot shows the estimations for time delays of $0.4 \mathrm{~s}, 0.8 \mathrm{~s}$ and $1.2 \mathrm{~s}$. This highlights the typical range of estimation in energy change that might occur. A method for determining the appropriate delay is proposed later.

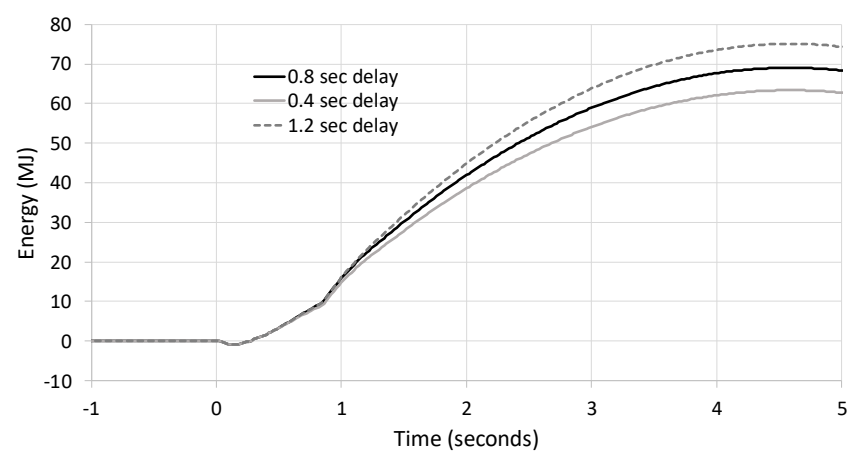

Fig. 5. Energy change caused by the perturbation for different delays in power system response

Frequency is calculated using a 25 sample moving window. This 0.5 second window is chosen to reduce the effect of measurement noise and system-wide frequency oscillation. Frequency values are returned for each 0.2 second increment, starting from $t=-0.3 \mathrm{~s}$. The window is centered on these values, thus the frequency at $t=-0.3 \mathrm{~s}$ is not affected by the perturbation and provides the pre-perturbation frequency. By taking the frequency deviation between two points in time and the appropriate change in energy, a value of kinetic energy for the power system inertia can be calculated. This results in a large set of inertia estimations, one for each combination of start and end point. An illustrative example is presented in Fig. 6, which shows how the start and end points relate to $\Delta E$ and $\Delta f$ for use in (8) to calculate a 'two-point' estimate. Fig. 6 also shows the delay and linear slope used to estimate $P_{m}$. The delay can be varied as appropriate, whereas $\mathrm{t}=0$ and the frequency nadir represent fixed points on the $P_{m}$ characteristic. Fig. 7 shows the inertia estimations for the simulation case of $20000 \mathrm{MWs}$ inertia and $10 \%$ droop for three starting points; $t=-0.3 \mathrm{~s}, t=0.5 \mathrm{~s}$ and $t=1.1 \mathrm{~s}$. The markers indicate the end points of each twopoint estimation.

Shorter time periods, close to the start will be most affected by the impact of the rapid power change on the calculation of frequency, as observed in Fig. 2. In addition, time periods closer to the frequency nadir will be affected by error in the calculation of power system response and by background variation. Thus, it will be necessary to restrict the analysis to those inertia values deemed more accurate. The average of the values that are deemed appropriate for the analysis will result in the inertia estimation. Based on observation of the real data, start points in the range $-0.3 \mathrm{~s}$ to $0.9 \mathrm{~s}$ have been used, with start points between $0.5 \mathrm{~s}$ and $0.8 \mathrm{~s}$ neglected. End points in the range $1.9 \mathrm{~s}$ to $2.7 \mathrm{~s}$ have been applied. This results in estimation windows ranging from $1.1 \mathrm{~s}$ to $3 \mathrm{~s}$, providing 30 two-point system inertia estimates for each transient.

The standard deviation of the two-point inertia estimates is calculated and used to determine the overall data integrity for a particular transient and to identify suitable temporal regions for analysis. This assists in determining which start and end points are included in the analysis. The procedure aims to include as much data as possible, while minimizing the standard deviation of the two-point estimates.

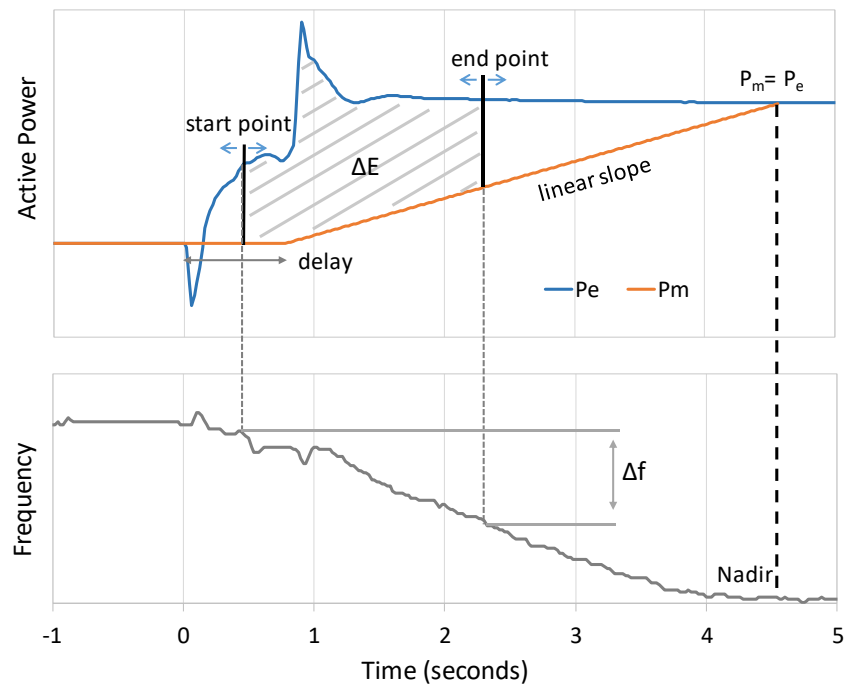

Fig. 6. Illustration of method for a two-point inertia estimate

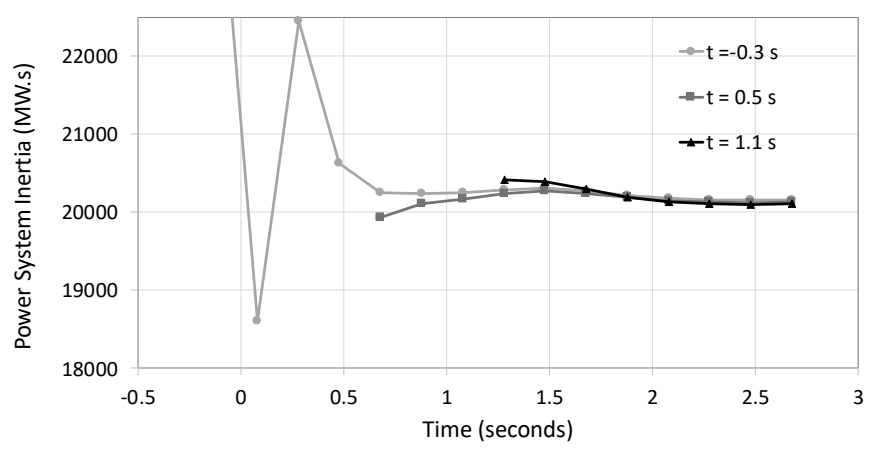

Fig. 7. Inertia estimated using simulated data, $20000 \mathrm{MWs,} 10 \%$ droop

The standard deviation of two-point estimates is also used to determine the appropriate time delay for the simple model of power system governor response and load relief. To achieve 
this, the procedure is repeated with delays ranging from $0 \mathrm{sec}-$ onds to 2.7 seconds, with the time delay resulting in the lowest standard deviation of the two-point inertia values then being used to estimate the inertia. The minimization of standard deviation approach has worked well with the simulation, getting within 0.1 seconds of the delay that gives the most accurate inertia estimation. For the simulated data, the average inertia estimation error for a system droop of $10 \%$ was $+0.76 \%$, a droop of $7.5 \%$ provided an average error of $+1.4 \%$, and a droop of $5 \%$ gave an average error of $+2.36 \%$.

By applying the technique to simulated data it is observed that the error in the estimated inertia will be higher when the power system has a low value of droop, i.e. when the power system responds more aggressively to the change in frequency. However, it will be observed that this is small compared to the variation in inertia estimates achieved from the real data.

The process for the inertia estimation methodology presented in this section is summarized by the flowchart in Fig. 8.

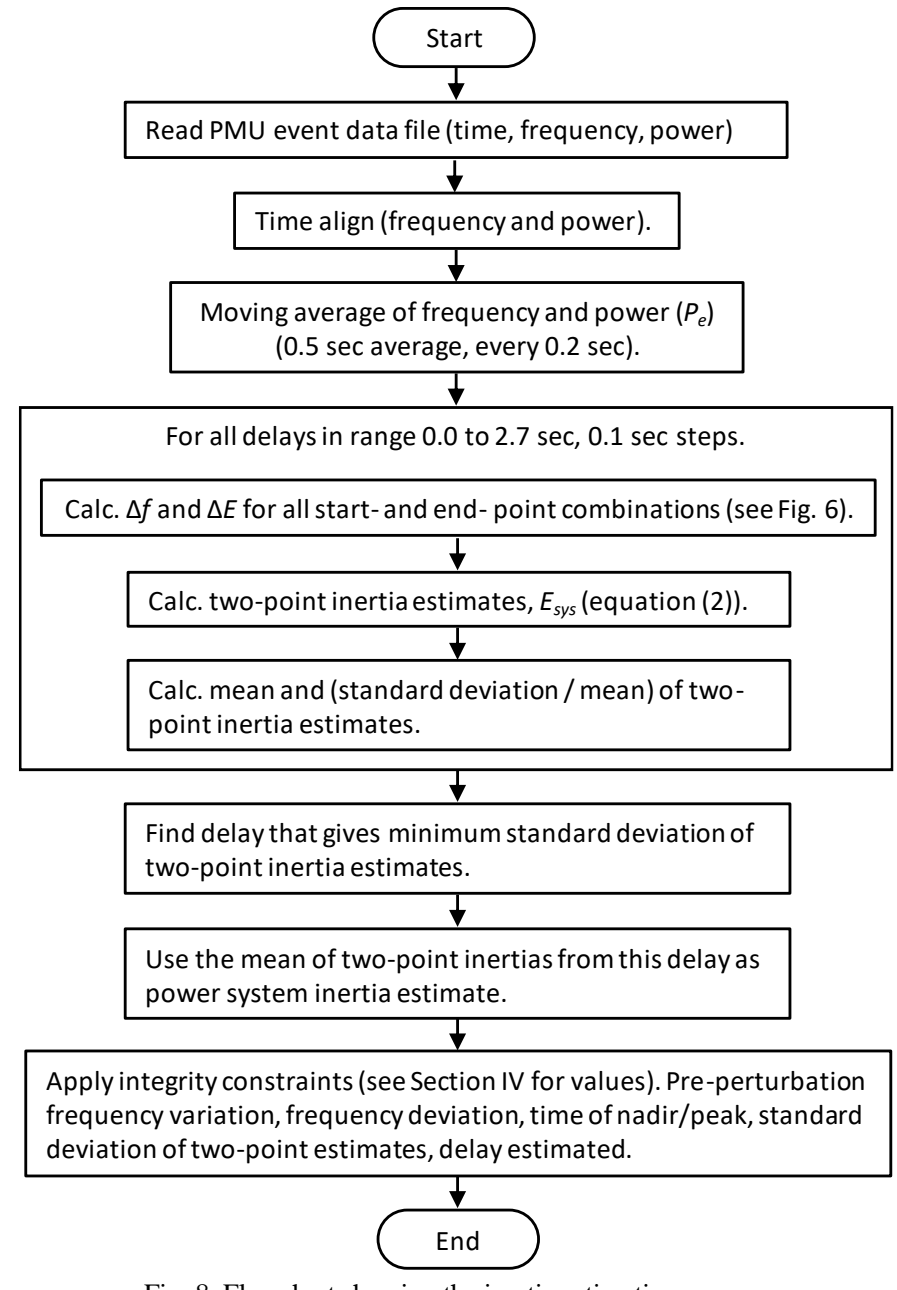

Fig. 8. Flowchart showing the inertia estimation process

\section{Data Preparation}

To complement the power perturbation data, information from the SEMO is also applied [21]. Half-hourly metered data from all the units on the network is available. The time of the perturbation is aligned with the half-hour period of data, and this allows parameters such as generation capacity, system demand and wind generation at the time of the perturbation to be determined. Synchronous generator inertia can also be estimated by using the system operator inertia values for each unit. The half-hour resolution of data means that if a particularly large unit is not actually synchronized at the time of perturbation, but still within the half-hour period that the perturbation occurs, errors potentially exceeding 3000 MWs could be present. To reduce this effect, the half-hourly periods immediately before and after are compared and if the synchronous generator inertia varies by more than $2000 \mathrm{MWs}$, these tests are eliminated from the data set. The synchronous generator inertia will account for most of the power system inertia and will be used as the principal validation metric for the analysis.

\section{APPLICATION TO REAL POWER SYSTEM DATA}

The technique is now applied to the real power system data. If the time delay for minimizing the standard deviation is very large or small, it is considered that background variation of frequency during the event has been too severe. Thus, if the minimum is not achieved by a delay in the range $0.2 \mathrm{~s}$ to $2.6 \mathrm{~s}$ the event is removed from the analysis. For the same reason, data where the pre-perturbation frequency varies by more than $0.015 \mathrm{~Hz}$ in the $1.5 \mathrm{sec}$ before the event are ignored.

In addition, if the minimum value of standard deviation $>8 \%$ of the estimated inertia, it is deemed unreliable. Data is also removed if the frequency deviation is very small, $<0.025 \mathrm{~Hz}$, the frequency nadir or peak occurs within $3.4 \mathrm{~s}$, or if the frequency nadir or peak does not occur in the captured data at all. The aim is to remove data that have been severely affected by background variation of frequency or instances where insufficient data have been captured. These integrity constraints leave 750 events, out of 1200, for further analysis.

From the remaining data, the average power system inertia is $40100 \mathrm{MWs}$, with an average two-point inertia standard deviation of 1100 MWs. The scatterplot in Fig. 9 shows the power system inertia estimates plotted against the synchronous generator inertia for the 750 events analyzed. The average synchronous generator inertia is $29800 \mathrm{MWs}$. For the whole year, of 2015, the average synchronous generator inertia was 30730 MWs. The value in 2010 was 37600 MWs, with an estimate of 28300 MWs for 2020 [1], indicating a trend of reducing inertia.

The analysis shows that on average the synchronous machine inertia accounts for $74.3 \%$ of the estimated power system inertia. The synchronous generator inertia data should be reasonably accurate, although errors may be present in the information provided to the system operator. The trend line in Fig. 9 allows an initial comparison to be made between power system inertia and synchronous generator inertia. The trend line appears to suggest that on average there is a base inertia of $7320 \mathrm{MWs}$ and that the increment in power system inertia is 1.1 times that of the synchronous generators' declared value. However, as will be shown, the relationship cannot be fully explained in such a simplified manner. Three other sources of inertia will be considered; system loads, other generation, and power plant auxiliary loads. 


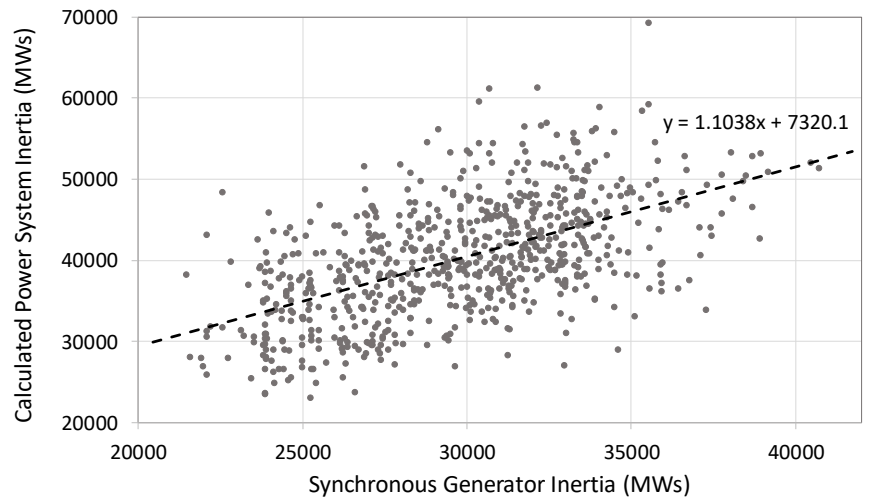

Fig. 9. Power system inertia versus synchronous generator inertia

\section{A. Inertia response from power station auxiliary loads}

The inertia contribution from power station auxiliary load will be explored through an example and then applied generally to the whole system. A PMU has been installed at a $220 \mathrm{MW}$ power station measuring both the synchronous generator and $11 \mathrm{kV}$ site load. Several responses to frequency disturbances caused by large generators have been observed. Fig. 10a is an example showing the frequency transient, dropping to $49.3 \mathrm{~Hz}$, and voltage fluctuation on the $11 \mathrm{kV}$ network. Fig 10b shows the response from the synchronous generator and the site load. The inertial response from the synchronous generator can clearly be observed during the first 2 seconds of the transient, the unit then provides primary operating reserve based on a droop response.

The response from the site load is interesting, with the peak power response being $9.3 \%$ of the synchronous generator's peak inertial response. As well as an inertia contribution from motors the load will also provide load relief if frequency and voltage are depressed. However, the load does not appear to show strong correlation with the relatively small voltage fluctuation. In addition, the peak occurs well before the frequency nadir, thus it can be considered that most of the peak in response is due to the motors' inertial response. The power station has many large asynchronous motors, such as the $4.6 \mathrm{MW}$ boiler feedwater pumps. The peak of the load response occurs $300 \mathrm{~ms}$ after the synchronous generator, which is a combination of how an induction machine's torque-speed characteristic is affected by the dynamic grid frequency, i.e. the slip, and the reasonably high inertia of these motors. Thus the site load, being predominantly induction machines, does not provide 'synchronous inertia' in its strict sense, but does provide an inertial response by the release of rotational kinetic energy. Over several observations, the average peak site load response was $8.6 \%$ of the synchronous generator. In terms of energy, after 1.0 second the site load inertial energy response is $7.6 \%$ that of the synchronous machine. But after 1.5 seconds this has risen to $9.2 \%$, and after 2 seconds it is $10.0 \%$. The rise is caused by the differing inertial response between synchronous and asynchronous machines.

This poses an interesting aside, in that the synchronous inertia of synchronous generators is being remunerated as part of Ireland's new system services [2]. Should the site load inertial response of power stations also be remunerated? It would seem that this provides a significant part of the power system's inertia, potentially several 1000 's of MWs. This would act as an incentive for new power plant to use grid connected motors, rather than interfacing through power electronic drives that remove the motors' inertial contribution.

Based on Fig. 10b, the inertia constant of the site load, using a base of the site load in MW, is approximately the same as that of the synchronous generator, using the MW capacity as the base. For the purposes of this work the site load inertia will be referenced to the power plant capacity rather than the synchronous generator's inertia. This would suggest that site load provides 0.5 to $0.6 \mathrm{MWs}$ per MW of synchronous generator capacity, adding approximately $10 \%$ to a typical power station's inertia contribution.

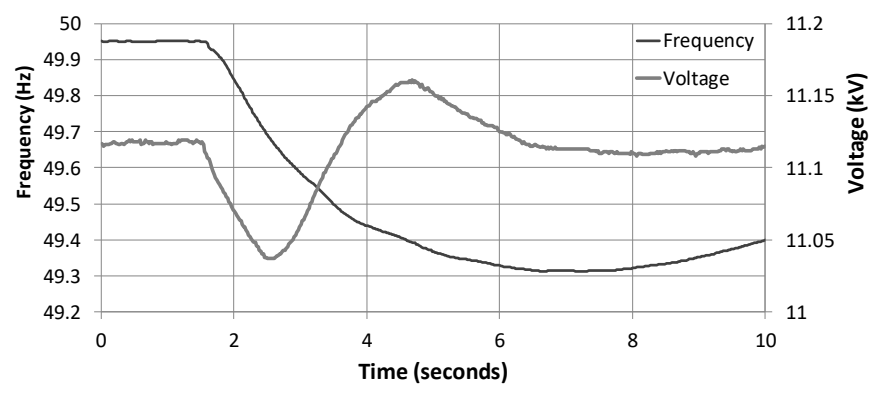

a

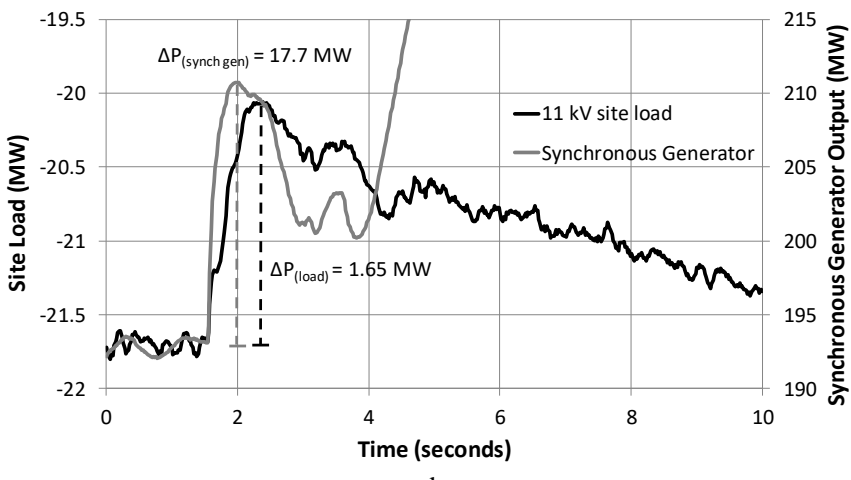

b

Fig. 10. a) Frequency transient and voltage fluctuation, b) Power response from synchronous generator and site load

\section{B. Inertia from other generators, including wind turbines}

There are several fixed-speed induction generator wind farms in Ireland, with approximately $240 \mathrm{MW}$ of capacity. In addition, a high proportion of the small scale wind sites, particularly in Northern Ireland, are likely to be fixed-speed wind turbines, bringing the capacity of this technology to perhaps 300 MW. Based on the inertia constant calculated in [22] for this type of wind turbine, they should contribute approximately 1200 MWs when connected to the grid.

Data available from [23] and [24] indicate that around 300 MW of synchronously connected distributed generation was present during the data collection period. A reasonable assumption would be for fixed-speed wind and synchronous distributed generation to contribute $1500 \mathrm{MWs}$ to $2000 \mathrm{MWs}$.

\section{Inertia from load demand}

The inertia contribution of load will be difficult to determine, but may account for much of the remaining power system inertia. A relevant study on the GB power system [5] indicated that the demand side would contribute $17 \%$ to $25 \%$ of the power 
system inertia with and average load inertia constant of $1.75 \mathrm{~s}$ that is higher at night and lower during the weekend.

The demand inertia constant, $H_{d e m}$, can be estimated from (9), where $E_{S G}$ is the kinetic energy of synchronous generation (MWs), $\mathrm{S}_{\mathrm{SG}}$ is the dispatched MW capacity of synchronous generation, $\alpha$ is the incremental power system inertia of power station auxiliary load per MW capacity, $\mathrm{E}_{\mathrm{DG}}$ is the kinetic energy of distributed generation and fixed speed wind, and $\mathrm{P}_{\mathrm{dem}}$ is the system demand (MW). Equation (9) is applied in the next section using grouped data to make several observations.

$$
H_{d e m}=\left(E_{s y s}-E_{S G}-\alpha \cdot S_{S G}-E_{D G}\right) / P_{d e m}
$$

\section{Time-of-day analysis}

The large number of perturbations permits variation in system conditions to be analyzed. A time-of day analysis is performed with the events grouped by the $1 / 2$ hour period in which they occurred. As the events relate to changes in power flow on HVDC interconnection they are most numerous during the main generation transition periods with $33 \%$ occurring over the four hours between 06:00-08:00 and 21:30-23:30. In contrast, only $11 \%$ of events occur from 10:00-15:00. Nevertheless, time-of-day classification shows some interesting features.

Fig. 11 shows a 2.5 hour moving window of load demand inertia constant, calculated using (9), and system demand. Fig. 12 shows the time-of-day variation in power system inertia and the synchronous generator inertia.

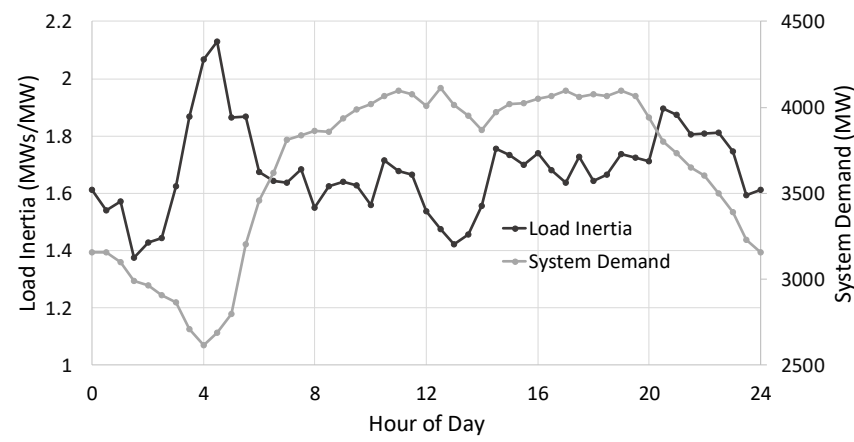

Fig. 11. Time-of-day variation of load demand inertia and system demand

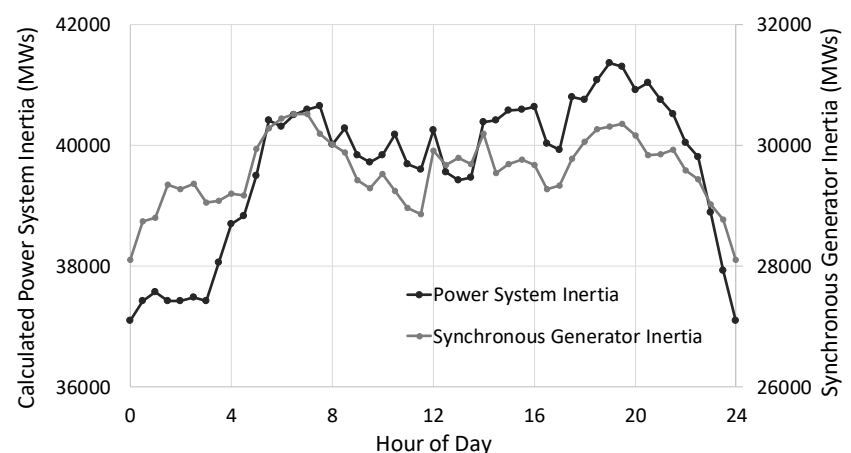

Fig. 12. Time-of-day variation of power system inertia and synchronous generator inertia

The inertia constant of load demand rises rapidly between 02:30 and 04:00, several hours before the demand. This is also where minimum demand occurs. Several factors may play a part. For example, permanently connected motors forming the base load may effectively increase the load inertia constant at low demand, or certain industrial process may make use of lower nighttime tariffs. However, the most likely cause is the start-up of bulk generation, with an increase in power plant motor load, and perhaps also including the pre-generation synchronization of the units, several hours before generation starts. Start of generation from the additional units committed for daytime demand typically occurs from 04:30 to 05:30, which can be observed as a rise of synchronous generator inertia during this period in Fig. 12.

As a result, although the minimum power system inertia occurs during the night it is several hours before the minimum demand. The disconnection of bulk generation in the evening, while power plant site load inertia remains high during shutdown procedures would offer explanation of the slightly higher load demand inertia constant in the evening.

\section{E. Effect of Wind generation and Weekday-Weekend}

Further analysis will investigate the effect of wind generation and compare weekdays with weekends. However, with each additional division fewer events will be available. There were not enough events captured during the winter, particularly around evening peak, to extend the analysis to seasonal variation. However, by restricting analysis to the summer and bordering months, April to September, an analysis of wind generation is made possible. The reason for the seasonal restriction when considering wind variation is that low wind generation in winter is associated with low temperatures and thus higher demand. This is not the case in summer due to Ireland's climate and the relatively small amount of cooling load on the network. In addition, the demand variation is less in summer and so this has less influence on the analysis.

The 'summer' events are separated into high- and low-wind generation and into four different time-periods in Fig. 13 and Fig. 14. The 2330-0300 period relates to minimum power system inertia, 0330-0530 represents minimum demand, 06002000 has relatively stable average demand and inertia, and 2030-2300 represents the evening transition period. A weekday-weekend comparison is only possible during the 0600-2000 time-period. 'High-wind' has an average generation of 1200 MW, while the 'low-wind' average is $400 \mathrm{MW}$.

It can be observed in Fig. 13 that system inertia is higher at times of low wind, by 3000 to $6000 \mathrm{MWs}$ on average. The lowest power system inertia occurs during the 2030-0300 high wind scenario, an average of 34000 MWs. Weekend daytime power system inertia is lower than weekday inertia by 3000 to 4000 MWs on average. This corresponds to a daytime demand that is lower on weekends by $500 \mathrm{MW}$ on average.

The demand inertia constant is estimated using (9) and shown in Fig. 14. The increase of this value during the 03300530 period is evident, as previously observed in Fig. 11, and assumed to be primarily due to the startup of synchronous generation. The slightly higher demand inertia constant in the evening 2030-2300 is also observed, which may similarly be caused by the shutdown of power plant. Thus, taking these factors into consideration, the inertia constant of the system demand may not vary noticeably and lies in the range 1.4 to $1.6 \mathrm{MWs} / \mathrm{MW}$.

While the load demand inertia constant in Fig. 14 appears to increase when wind generation is higher, this is likely to be a 
residual effect from synchronous generator commitment. It is most pronounced during the $0330-0530$ period, but also evident during the daytime-period. This may be a consequence of the increased flexibility of conventional generation required during high wind. The average three-hour variation of wind generation in the high-wind scenario is $200-300 \mathrm{MW}$ compared to 100 $150 \mathrm{MW}$ for low-wind. While, in the daytime period, the threehour variation in synchronous inertia is $350-400 \mathrm{MWs}$ more during high-wind. Thus, when wind output is more variable, the supporting synchronous plant is more likely to be in a non-generating inertia-providing state, resulting in a perceived increase in demand inertia constant. There is no clear difference in the demand inertia constant between weekend and weekdays.

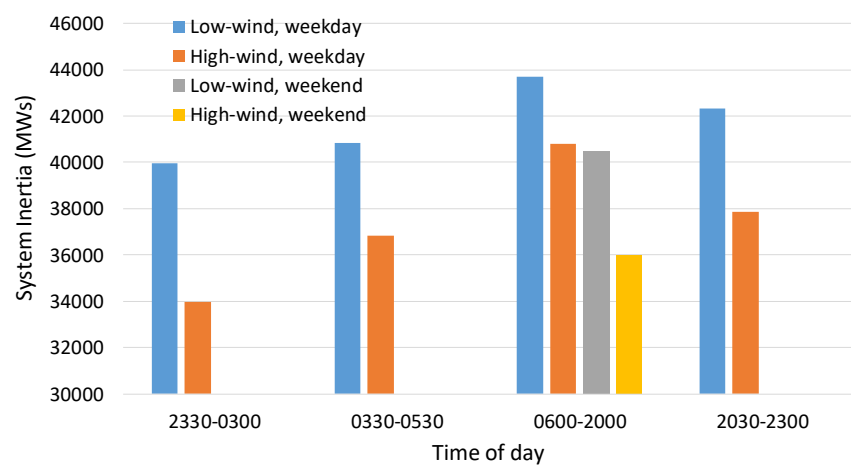

Fig. 13. Power system inertia; wind generation and weekday-weekend comparison

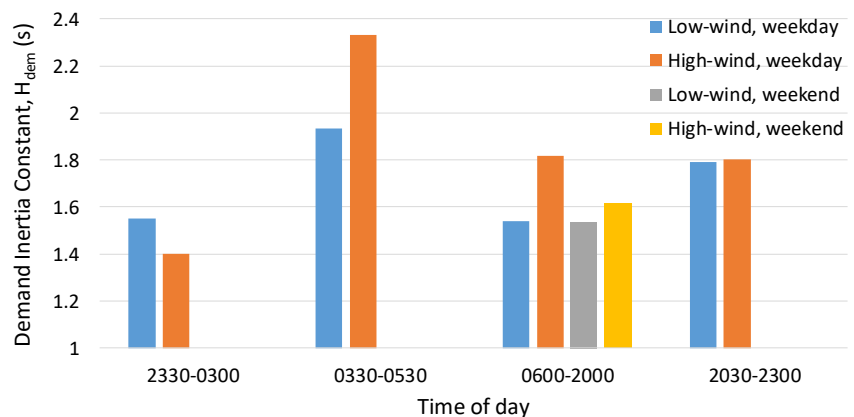

Fig. 14. Load demand inertia constant; wind generation and weekday-weekend comparison

\section{IMPROVEMENTS}

A practical implementation might employ a 'multiple-shot' technique, whereby several perturbations are performed in succession at time intervals that permit the system to recover in between, for example perturbations over the course of a few minutes. This means an average value of inertia could be determined and outliers could be more easily eliminated, thus improving the accuracy of the inertia estimate. The largest disturbance that is acceptable to be repeated many times will provide the best estimation results from this technique.

Further improvement could be achieved via the frequency measurement. As previously mentioned, a globally calculated frequency from several sites would reduce the effect of noise, local effects and inter area oscillation on the determination of inertia. This would require additional PMU infrastructure and communications, but these devices are now widely installed, even if their full potential to benefit power system operation has not yet been realized. The use of global frequency would also allow the data moving average window to be reduced and the overall inertia estimation window to be extended, permitting more of the transient to be used for inertia estimation.

A further step would be to replace the power system frequency measurements with machine speed measurements from rotary encoders, thereby allowing data averaging windows to be minimized. This would improve the resolution of inertia estimation and may be a necessity in smaller systems or those with very low inertia. The speed measurements could be taken directly from bulk generators, or via standardized synchronous motors acting as speed measurement devices at various parts of the network. In addition, testing inertia using perturbations at multiple locations on the grid could also serve to refine the inertia estimation. Validation of the method will be aided by the enhancements discussed in this section, supported by operational experience and comparison with existing, but less ideal, techniques.

It is worth noting that this power perturbation affects two power systems, both Ireland and Great Britain, and so there is an opportunity to test two systems simultaneously. At present, a device has been commissioned in Great Britain [12] to measure power system inertia in a similar manner [20].

\section{CONCLUSION}

A power system inertia estimation technique has been developed using a Matlab simulation and real power system measurements. This is made possible by PMU monitored power perturbations that occur when an HVDC interconnector switches power flow. The method employs an energy based estimation technique that allows a power change, which is not a step response, to be used for inertia calculation. A balance is made between maximizing estimation accuracy and the various additional factors present in the real data that affect power system frequency and its measurement. The technique also accounts for differing power system response conditions, such as droop response and load characteristics. The estimation method has been applied to a data set of 1200 transients, which was reduced to 750 for further analysis by the integrity constraints.

As might be expected, it has been shown that synchronous generators account for some $75 \%$ of the power system inertia. This suggests a significant inertia contribution from distributed generation, power station auxiliary load and power system demand. Plant auxiliary load provides a valuable, potentially remunerable, source of inertia and is estimated as 0.5 to 0.6 MWs/MW power plant capacity, thus increasing the inertia contribution from a power station by approximately $10 \%$. The contribution from system demand is estimated to be 1.4 to 1.6 MWs/MW, with an additional 1500 to $2000 \mathrm{MWs}$ from fixedspeed wind and synchronous distributed generation.

Time-of-day analysis initially suggested that the demand inertia constant varies considerably and is most prominent several hours before the morning rise in demand. However, this perceived variation may not be due to the demand itself. One of the probable reasons for the variation is the connection of power plant auxiliary load and synchronous generators during the 
start-up process several hours before generation occurs. The result is that minimum power system inertia occurs several hours before minimum demand. A similar effect is also observed in the evening and may be attributed to power plant shut down procedures. Further analysis suggests that the requirement for additional flexibility from conventional plant with increasing wind penetration means that more time is spent in a non-generating, inertia providing state.

In order to adapt the method for practical implementation several modifications could be applied. Frequency could be captured from multiple dispersed locations or complemented by machine speed measurements, and the use of a multiple-shot perturbation would help eliminate obvious outliers. The power perturbation could be provided by any suitable source that has sufficient capacity and controllability. For example, both battery energy storage systems and HVDC interconnections are capable of this.

The contribution of inertia from sources other than bulk synchronous generation will be increasingly important in power systems which operate with low inertia levels due to high amounts of non-synchronous infeed. The method can be extended to determining other factors, such as the system response characteristics. This knowledge of inertia and system conditions could then be directly fed into updatable controllers of energy storage inverters being used for grid stability, operating reserve, and synthetic inertia provision to improve their value to the power system.

\section{REFERENCES}

[1] EirGrid, SONI "Ensuring a Secure, Reliable and Efficient Power System in a Changing Environment", Jun. 2011 [Online]. Available: www.eirgridgroup.com

[2] Eirgrid Group. DS3 Programme. [Online]. Available: http://www.eirgridgroup.com/how-the-grid-works/ds3-programme

[3] P. V. Brogan, R. J. Best, D. J. Morrow, R. Duncan, M. L. Kubik, "Stacking battery energy storage revenues with enhanced service provision", IET Smart Grid, in press.

[4] P. M. Ashton, C. S. Saunders, G. A. Taylor, A. M. Carter, M. E. Bradley, "Inertia estimation of the GB power system using synchrophasor measurements", IEEE Trans. Power Syst., vol. 30, no. 2, pp. 701-709, Mar. 2015.

[5] Y. Bain, H. Wyman-Pain, F. Li, R. Bhakar, S. Mishra, N. P. Padhy, "Demand side contributions for system inertia in the GB power system", IEEE Trans. Power Syst., vol. 33, no. 4, pp. 3521-3530, Jul. 2018

[6] I. HR, R.J. Best, D.J. Morrow, "Irish power system primary frequency response metrics during different non synchronous penetration", in Proc. 2015 IEEE PowerTech, pp. 1-6

[7] M. R. B. Tavakoli, M. Power, L. Ruttledge, D. Flynn, "Load inertia estimation using white and grey-box estimators for power systems with high wind penetration", in IFAC Proc., $8^{\text {th }}$ Power Power Plant and Power System Control Symposium, vol. 45, no. 21, pp. 399-404

[8] D. Zografos, M. Ghandhari, "Power system inertia estimation by approaching load power change after a disturbance", in Proc. 2017 IEEE Power \& Energy Society General Meeting, pp. 1-5

[9] P. Wall, V. Terzija, "Simultaneous estimation of the time of disturbance and inertia in power systems", IEEE Trans. Power Deliv., vol. 29, no. 4, pp. 2018-2030, Aug. 2014

[10] K. Tuttelberg, J. Kilter, D. Wilson, K. Uhlen, "Estimation of power system inertia from ambient wide area measurements" IEEE Trans. Power Syst, vol. 33, no. 6, pp. 7249-7257, Nov. 2018

[11] A. Schmitt, B. Lee, "Steady-state inertia estimation using a neural network approach with modal information", in Proc. 2017 IEEE Power \& Energy Society General Meeting, pp. 1-5.

[12] Hosaka, Naoki, Brian Berry, and Satoshi Miyazaki. "The World's First Small Power Modulation Injection Approach for Inertia Estimation and Demonstration in the Island Grid." In 2019 8th International Conference on Renewable Energy Research and Applications (ICRERA), pp. 722726. IEEE, 2019.

[13] F. Allella, E. Chiodo, G. M. Giannuzzi, D. Lauria and F. Mottola, "OnLine Estimation Assessment of Power Systems Inertia With High Penetration of Renewable Generation," in IEEE Access, vol. 8, pp. 6268962697, 2020, doi: 10.1109/ACCESS.2020.2983877.

[14] A. Fernández-Guillamón, A. Vigueras-Rodríguez and Á. Molina-García, "Analysis of power system inertia estimation in high wind power plant integration scenarios," in IET Renewable Power Generation, vol. 13, no. 15, pp. 2807-2816, 1811 2019, doi: 10.1049/iet-rpg.2019.0220.

[15] Yazdi, S.S.H., Milimonfared, J., Fathi, S.H., Rouzbehi, K. and Rakhshani, E., 2019. Analytical modeling and inertia estimation of VSG-controlled Type 4 WTGs: Power system frequency response investigation. International Journal of Electrical Power \& Energy Systems, 107, pp.446-461.

[16] C. Phurailatpam, Z. H. Rather, B. Bahrani and S. Doolla, "MeasurementBased Estimation of Inertia in AC Microgrids," in IEEE Transactions on Sustainable Energy, vol. 11, no. 3, pp. 1975-1984, July 2020, doi: 10.1109/TSTE.2019.2948224.

[17] P. V. Brogan, R. Best, D. J. Morrow, C. Bradley, M. Rafferty, M. Kubik, "Triggering BESS inertial response with synchronous machine measurements", in Proc. 2018 IEEE Power \& Energy Society General Meeting, pp. $1-5$

[18] EirGrid/SONI. Operational Constraints Update 27/01/2016. [online] available: www.eirgridgroup.com

[19] P. V. Brogan, R. J. Best, D. J. Morrow, K. McKinley, M. L. Kubik, "Effect of BESS response on frequency and RoCoF during underfrequency transients", IEEE Trans. Power Syst, vol. 34, no. 1, pp. 575-583, Jan. 2019

[20] J. Zhao, Y. Tang, V. Terzija, "Robust online estimation of power system center of inertia frequency", IEEE Trans. Power Syst, vol. 34, no. 1, pp. 821-825, Nov. 2018

[21] Single Electricty Market Operator, http://www.sem-o.com

[22] R. J. Best, D. J. Morrow, "Analysis of two-speed wind farm operation from grid-side measurements", IEEE Trans. Sust. Energy, vol. 4, no. 3 , pp. 689-697, Jul. 2013

[23] Renewable Energy Foundation, http://www.ref.org.uk

[24] ESB Networks, Distribution Connected-energised Non-Wind [Online]. available: https://www.esbnetworks.ie 